\title{
Asynchronous Teleconsultations: The US Military Experience in the Pacific
}

\author{
Jennifer MBUTHIA ${ }^{\mathrm{a}, \mathrm{b}, \mathrm{c}, 1}$ and Mechelle MILLER ${ }^{\mathrm{d}}$ \\ ${ }^{a}$ Colonel, Retired, US Army Medical Corps, Honolulu, Hawaii \\ ${ }^{\mathrm{b}}$ Assistant Professor of Pediatrics, Uniformed Services University of the Health \\ Sciences, Bethesda, Maryland \\ ${ }^{\mathrm{c}}$ Clinical Informaticist, Queens Medical Center, Honolulu, Hawaii \\ ${ }^{\mathrm{d}}$ Major, US Army Medical Corps, Tripler Army Medical Center, Department of \\ Pediatrics, Honolulu, Hawaii
}

\begin{abstract}
Tripler Army Medical Center (TAMC), located in Honolulu, Hawaii, serves as the US military's tertiary medical referral center for the Western Pacific. Over 20 years ago, the TAMC Department of Pediatrics developed an asynchronous provider-to-provider teleconsultation pilot program, eventually named the Pacific Asynchronous TeleHealth (PATH) system. A secure teleconsultation platform for pediatric sub-specialty provider-to-provider advice, the platform grew based on the needs of users, eventually expanding to serve all age-groups, with over 60 different specialties based at TAMC providing teleconsultation. Eventually, the success of PATH drove further expansion to serve military clinicians located in other overseas locations beyond the Asia-Pacific. This cost-effective model can be applied to civilian healthcare settings, particularly where geographic distance or limited connectivity are challenges to delivery of synchronous telehealth or in-person specialty care.
\end{abstract}

Keywords. Asynchronous, teleconsultation, store-and-forward, military, Pacific Region

\section{Introduction}

The US Department of Defense (DoD) Military Health System provides medical care to military Service Members and their family members around the globe. Tripler Army Medical Center (TAMC), located in Hawaii, is the tertiary medical referral center serving the DoD beneficiaries throughout the Western Pacific Region. This footprint includes more than 20 military clinics and small hospitals on installations located in Guam, South Korea, and Japan (Figure 1). The wide geographic span of this region across large bodies of water is also the only military region that spans the International Date Line, and involves 5 different time zones. Routine, in-person evaluation by a subspecialist located at TAMC involves air travel and several nights of lodging, making the utilization of telemedicine a more cost-effective tool for routine ambulatory subspecialty evaluations.

Coordination of synchronous telemedicine visits between TAMC and facilities located in Japan, Guam, and South Korea can be very challenging and disruptive to the

${ }^{1}$ Corresponding Author: Jennifer Mbuthia, Uniformed Services University of the Health Sciences, Maryland. Queens Medical Center, Hawaii; Email: Jennifer.Mbuthia@gmail.com 
daily workflow of the distant site provider. Provider-to-provider synchronous discussions regarding routine medical questions about a patient can also be an interruption to daily work for both the requesting primary care clinician and the subspecialist. Alignment of schedules, challenging and/or complicated processes for coordination of appointments, requirements for video equipment and a secure platform, and the bandwidth requirements for a synchronous video encounter are all barriers to gaining buy-in from healthcare providers and from patients.

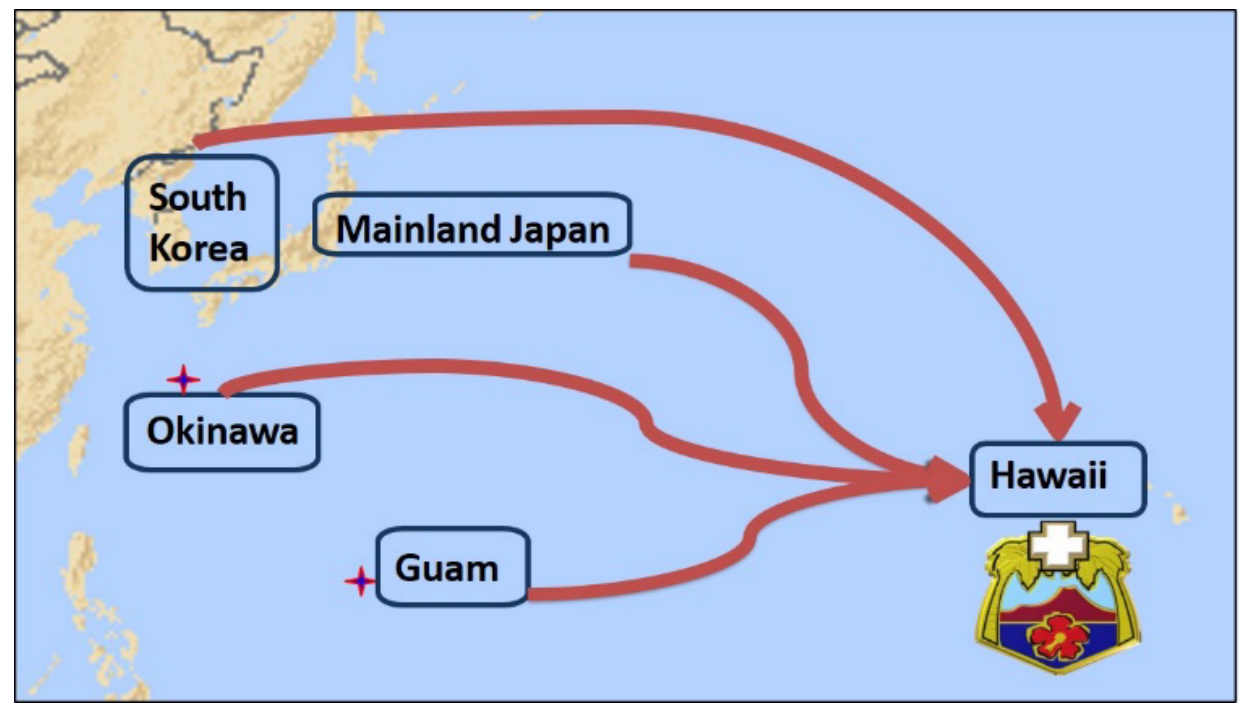

Figure 1. A map of the footprint where TAMC and PATH provide teleconsultation support. The locations highlighted above include military hospitals and clinics where $>95 \%$ of referrals originate. Additional referrals come from locations throughout the Asia-Pacific in locations where Active Duty military and their family members are stationed, such as Australia, Singapore, and Thailand.

\section{The Electronic Children's Hospital of the Pacific (ECHO-Pac)}

In search of a way to provide subspecialty support to primary care clinicians stationed in the Western Pacific Region, the TAMC Department of Pediatrics received funding to establish a demonstration project utilizing a secure, asynchronous (store-and-forward), web-based platform for provider-to-provider pediatric specialty teleconsultation [1]. Initially called the Electronic Children's Hospital of the Pacific (ECHO-Pac), a webbased platform was built that would be compliant with the Health Insurance Portability and Accountability (HIPAA) Act and security requirements of a web-based healthcare platform. ECHO-Pac was initially used to provide support by pediatric pulmonologists at TAMC to 7 children with moderate to severe asthma (mean age $11.9+/-3.7$ years) who were military dependents and living overseas in the Pacific Region [2]. It served as a way to augment the care they received from their general pediatrician or other primary care manager. Video recordings of the patient using their metered-dose inhaler (MDI) were submitted to the pediatric pulmonologist at specified intervals, and reviewed regarding their technique. In this demonstration project, children with asthma followed by teleconsultation for 1 year had fewer emergency room visits for asthma $(3.85+/-5.14$, 
range $0-15$ vs. 0 visits, $\mathrm{p}<0.05)$ and fewer unscheduled acute clinic visits $(1.57+/-1.27$, range $0-4$ vs. $0.286+/-0.48, \mathrm{p}<0.05)$ in the study year versus the preceding year. There were two hospitalizations in the year prior to the study; however, no patients were hospitalized during the study.

\subsection{Evaluation of Asynchronous Teleconsultation on Care and Cost Avoidance}

Building upon the initial success with pediatric pulmonology and asthma consults, the Department of Pediatrics expanded the specialties available in ECHO-Pac, and the utilization of the system was systematically studied for a year to further characterize its use, impact on access to specialty care, and whether it prevented travel to Hawaii for routine outpatient specialty evaluation [1]. This study model added the role of consult managers at hub location, added in email notification to spoke and hub providers when new comments were added to a case (devoid of any patient-identifying information), and included the use of electronic backfill so that if a specialty provider at TAMC was away on extended leave, or was deployed and therefore unable to reply to teleconsultations, a pediatric specialist located at a different DoD tertiary care center was invited to reply to clinical teleconsultations to avoid underlap in coverage. Data was collected between 2002 and 2003, and included a total of 267 cases from 16 spoke locations in the AsiaPacific. A panel of 5 pediatric physicians who were not involved with the teleconsultations independently reviewed each case using a Likert scale to answer the following questions:

- Was the diagnosis changed or modified by the consultation?

- Was the diagnostic plan changed or modified by the consultation?

- Was the treatment plan changed or modified by the consultation?

Cost savings was evaluated by the physician review panel based on responses to the following question:

- If telemedicine were unavailable, would this patient have been evacuated on a routine flight to the medical center for consultation and care?

The diagnosis was changed or modified following the use of a teleconsultation in ECHO-Pac in $15 \%$ of cases, the diagnostic plan for evaluation changed in $21 \%$ of cases, and the treatment plan was changed or modified in $24 \%$ of cases $(\mathrm{p}<0.01)$. In addition, the review panel identified avoided patient movement in $12 \%$ of the cases, which was estimated to be a cost savings of approximately $\$ 185,408$ (USD) based on flight cost for one parent plus the child and the lodging and per diem for a 10 day stay.

\section{The Pacific Asynchronous TeleHealth (PATH) Program}

Over a 2 year period, the number of ECHO-Pac users at spoke locations increased, and more specialty providers at TAMC were requesting accounts, often finding it easier to reply to clinical questions using the asynchronous platform instead of receiving telephone messages and then trying to reach the asking clinician who might be in Japan, Guam, or South Korea. The success of the original demonstration project led to the renaming of the program to the Pacific Asynchronous TeleHeath (PATH) System in 2004. 


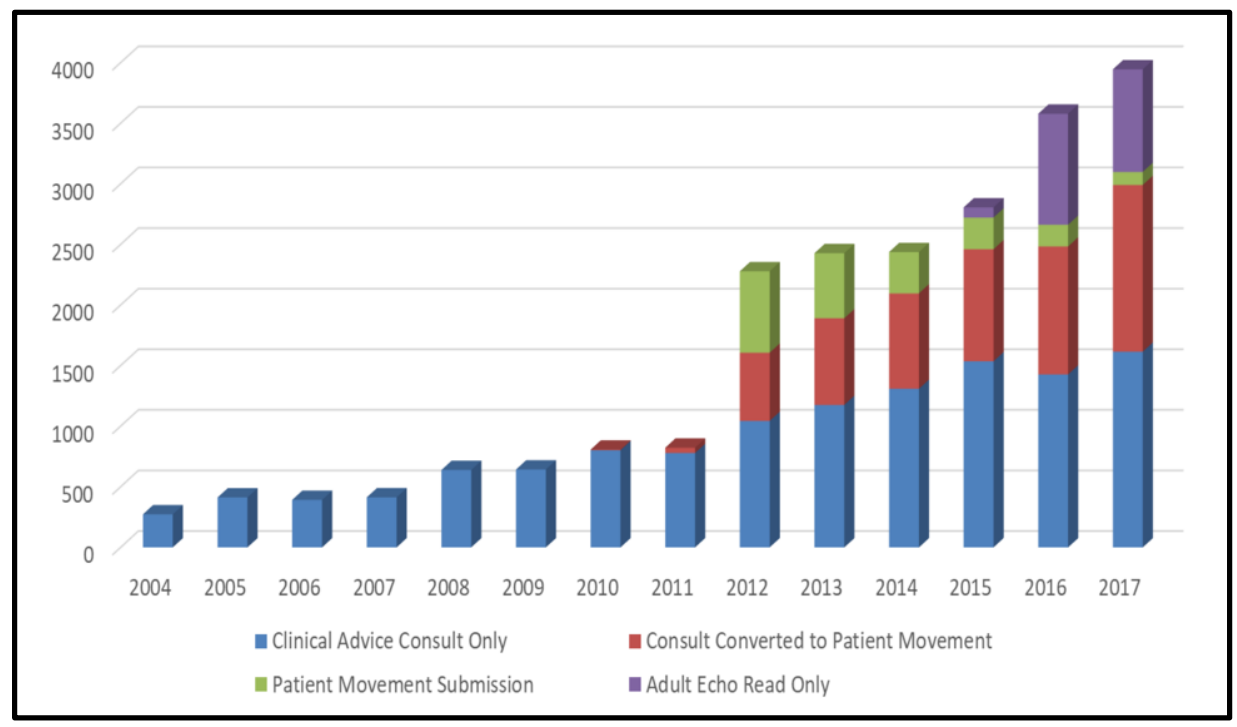

Figure 2. The Growth in Pacific Asynchronous TeleHealth (PATH): data showing the number of cases submitted from 2004 to 2017. The graph distinguishes between advice-only, patient movement to Hawaii, teleconsults that resulted in patient movement, and adult tele-echocardiogram requests.

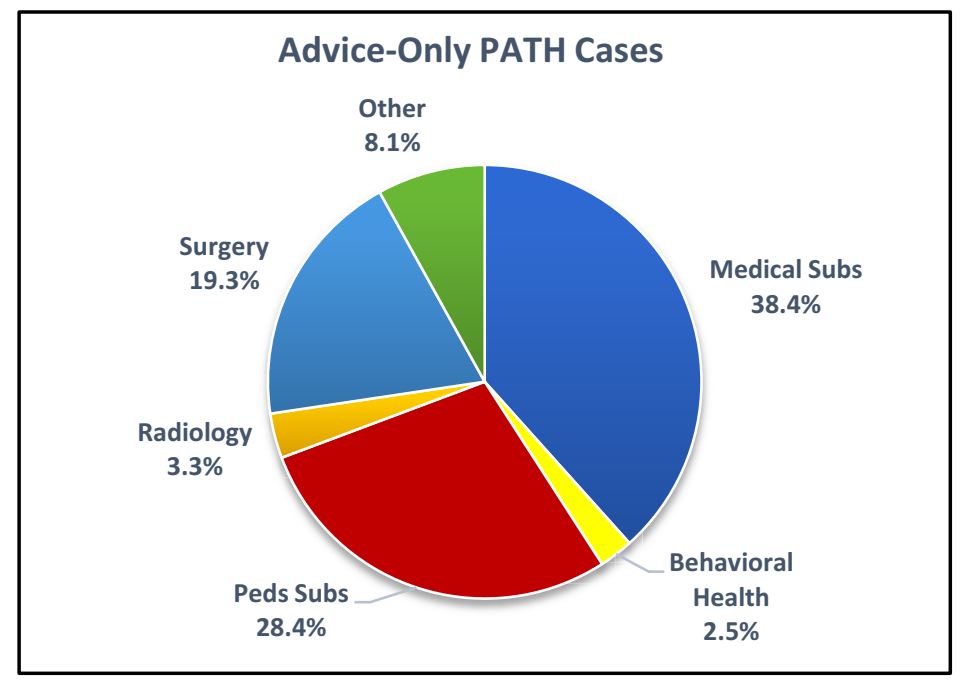

Figure 3. PATH advice-only cases (asynchronous teleconsultation) distribution across general medical specialty areas: adult medicine specialties, pediatric specialties, surgical specialties, behavioral health, and radiology. The group "Other" includes areas such as occupational medicine, pain management, and audiology (data captured from 2017-2018 PATH case data).

PATH expanded to include all age groups in 2009 due to continued request from end users at the spokes and the hub specialty providers, and then in 2012 the role of the system underwent a significant expansion by serving as both a tool for asynchronous teleconsultation and to coordinate appointments for either synchronous teleconsults or 
in-person visits at TAMC. By adding this care coordination functionality, PATH was able to improve the hand-off of patients who were coming to Hawaii. Prior to the use of PATH, the originating site provider did not have visibility of when specialty appointments or ambulatory procedures were available at TAMC, and many times patients would arrive at TAMC without pre-scheduled appointments, creating additional length of stay while additional appointments were worked into a specialty clinic. If a patient required an outpatient appointment with more than one clinical specialty at TAMC, this could exponentially increase the length of stay for in-person routine specialty appointments.

Table 1. Pacific Asynchronous TeleHealth (PATH) Specialties Available for Teleconsultation at Tripler Army Medical Center (based on 2018-2019 referral data).

\begin{tabular}{|c|c|c|c|}
\hline Adult Specialties & Pediatric Specialties & Surgical Specialties & Other \\
\hline Allergy/Immunology & Adolescent Medicine & Bariatric & Behavioral Health \\
\hline Audiology & Allergy/Immunology & Breast Surgery & Chemical Addiction \\
\hline Cardiology & Cardiology & Cardiothoracic & Interventional Rad. \\
\hline Clinical Pharmacist & Developmental Peds & Colorectal Surgery & Musculoskeletal Rad \\
\hline Concussion Clinic & Dermatology & Endo-Urology & Neuropsychology \\
\hline Critical Care/Intensivist & Endocrinology & Facial/Reconstructive & Nuclear Medicine \\
\hline Dermatology & Gastroenterology & Foot/Ankle & Radiation Oncology \\
\hline Endocrinology & Genetic Counselor & Hand/Upper Extremity & \\
\hline Endocrine/Fertility & Hematology/Oncology & Head \& Neck & \\
\hline Flight Medicine & Infectious Disease & Joint/Orthopedic & \\
\hline Gastroenterology & Neonatology & Maxilofacial & \\
\hline Genetic Counselor & Nephrology & Neuro-Otology & \\
\hline Hematology/Oncology & Neurology & Neurosurgery & \\
\hline Hospitalist & Occupational Therapy & Ophthalmology & \\
\hline Infectious Disease & Pediatric Intensivist & Orthopedics & \\
\hline Nephrology & Pediatric Psychiatry & Otolaryngology & \\
\hline Neurology & Pediatric Psychology & Pediatric Otolaryng. & \\
\hline Nutrition & Pulmonology & Pediatric Orthopedic & \\
\hline Obstetrics/Gynecology & Physical Therapy & Pediatric Surgery & \\
\hline Pain Medicine & Pediatric Radiology & Pediatric Urology & \\
\hline Pathology & Rheumatology & Plastic Surgery & \\
\hline Perinatology & & Refractive Surgery & \\
\hline Pulmonology & & Spine & \\
\hline Rheumatology & & Surgical Oncology & \\
\hline Sleep Medicine & & Trauma & \\
\hline Speech/ Pathology & & Urology & \\
\hline Sports Medicine & & Urology Oncology & \\
\hline Urogynecology & & Vascular Surgery & \\
\hline Vaccine Healthcare & & & \\
\hline
\end{tabular}

In 2015, PATH became the platform through which asynchronous adult teleechocardiograms could be requested from the military hospitals on Guam and Okinawa, Japan. Both of these locations had radiology technicians who were trained in performing echocardiograms, and given clear parameters for clinical concern where they should immediately contact the patient's primary care manager and the cardiology service at TAMC. The addition of this routine service to both of these military hospitals further decreased the cost of regularly rotating a military cardiologist to these locations in order to perform routine, ambulatory echocardiograms. Figure 2 shows the growth in the volume of PATH cases from 2004 through 2017, showing that the two greatest changes to the volume were incorporating coordination for care if in-person evaluation in Hawaii was indicated and adult tele-echocardiogram requests. Figure 3 shows the distribution of 
the asynchronous provider-to-provider (advice-only) PATH cases, with adult medicine specialties capturing almost $40 \%$ of total case volume. The top five adult medicine specialties consulted in PATH for advice are: neurology, hematology/oncology, cardiology, gastroenterology, and endocrinology. For pediatric patients, the top five specialties consulted in PATH for advice are: pulmonology, cardiology, neurology, endocrinology, and gastroenterology.

As the demands on PATH grew, the need for a dedicated administrative team at TAMC also grew. In June 2018, then PATH medical director, COL Jennifer Mbuthia, stood up the Department of Virtual Health at TAMC, and the administrative management of PATH now fell under this new department, allowing for dedicated resources to continue to support this mission critical program for military medicine.

PATH continues to support approximately 1,500 active users, with over 4,000 cases submitted each year from around the Asia-Pacific, and over 60 different specialties available for teleconsultation, including surgical and radiology sub-specialties (Table 1).

\subsection{Workflow Design}

From the creation as ECHO-Pac, the asynchronous teleconsultation platform was designed to be low-bandwidth, and relatively intuitive to use. New users submit a request for an account using their military email address, and all requests are reviewed by the PATH team at TAMC to verify the requestor is a healthcare provider. If unable to verify the user, additional information may be requested. All PATH users have a unique username and password, and only need to have internet access in order to submit a new teleconsultation. The PATH homepage includes reminders that this is for routine teleconsultations only to mitigate the use of the platform for emergency medical advice that should be done over the telephone.

The design of PATH mirrors a hub-and-spoke model, with multiple spoke facilities submitting clinical cases that are all routed to the hub at TAMC. When a new case is initiated, minimum requirements include free text entry of clinical history and a clinical question being asked. The originating site provider can also attach images, scanned documents, or short video clips into the case if needed. Once submitted, the consult is given a unique case number, routed to the consult managers located at TAMC for review to verify information in free text is complete, then it is routed to the requested specialists. More than one specialist can be consulted on a case. Clinical comments are then provided, and any subsequent communication within the system follows along with discrete timestamped text boxes. To facilitate end-user acceptance of the system, the times are displayed in their local time, and an email notification is also sent using only the PATH case number to inform them that a user has uploaded a new comment. Figure 4 shows an overview of the workflow described above.

PATH consult managers maintain visibility of all cases, and they are notified via email twice weekly of all cases that have not had a clinical response from the distant site/hub providers. This provides an added layer of accountability to insure timely responses to the teleconsultation.

PATH added the ability to submit pediatric pulmonary function test results from facilities that had the equipment and trained technicians to perform lung function testing on children. This could be done with or without a clinical question, especially if it was 
part of an annual asthma assessment on a child with well-controlled asthma who was being appropriately managed by their primary care provider.

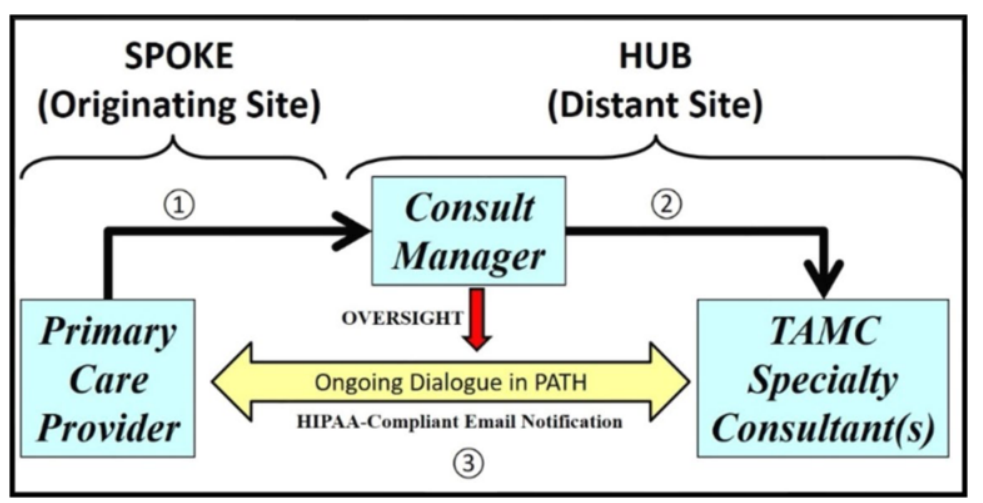

Figure 4. Overview of PATH workflow. (1) Case is created by clinician at the spoke, (2) reviewed by a PATH consult manager who forwards case to hub specialists (3)

\subsection{Evaluation of PATH Impact on Care and Cost Avoidance: 2006-2009}

A retrospective review of 1,000 pediatric teleconsultations from 2006 to 2009 demonstrated PATH system's positive impact with regard to access, quality, and cost, demonstrating the benefits of incorporating asynchronous provider-to-provider teleconsultation into routine pediatric practice [5]. Cases were reviewed by a panel of three pediatric providers, each independently completing a nine question survey after reviewing a case, and found that originating site providers achieved resolution of the clinical question $63 \%$ of the time. In addition, Mahnke et al found that the clinical question was looking for assistance in making a diagnosis in $72 \%$ of the cases, and asking questions regarding medical treatment in $21 \%$ of PATH cases reviewed.

\subsection{Evaluation of PATH Impact on Care and Cost Avoidance: 2017-2019}

As the clinical use of PATH continued to grow, assessing the impact it was having would be challenging if independent panels of reviewers were utilized. So in March 2017, the PATH system incorporated 2 survey questions for a user to reply to upon closing a case. The decision to limit the number of survey questions was rooted in finding a balance between obtaining data and end-users getting survey fatigue and not responding. Additionally, the responses gathered needed to have some meaning, or ability to link the responses or response trends to the value of the system. Value not only to the enduser/originating clinician, but also implied value to the patient by avoiding unnecessary travel and value to the organization by cost-avoidance [7]. The two questions were the following: (1) did this teleconsult prevented the need for patient movement? and (2) did this teleconsult recaptured care by preventing host nation/network referral?

In early 2020, TAMC Institutional Review Board (IRB) reviewed a research protocol to retrospectively review 3 years of responses to the two closing survey questions for all pediatric cases submitted. The consults assessed were submitted between March 2017 - February 2020, therefore the data did not include cases submitted 
after travel restrictions were implemented in the US due to the global pandemic from the severe acute respiratory syndrome-related coronavirus 2 , or SARS-CoV-2.

The study data has not been published at the time this chapter was written, but the initial evaluation of the data for pediatric subspecialty consultations was presented at National Telehealth Research Symposium in November 2020. The PATH system captured basic demographics, location, providers, subspecialists, and response to the 2 survey questions. De-identified data were obtained for 2,448 pediatric teleconsults during the study period. Fisher's exact tests and chi-squared tests were used to assess differences in survey response, preventing face to face visits and recapture care rates. For users in the Western Pacific, over $40 \%$ of cases had completed survey responses for pediatric specialty cases, and over $69 \%$ reported that the teleconsultation did prevent the originating primary care provider from requesting to send the patient to Hawaii for routine specialty evaluation. Utilizing the similar assumptions as Mahnke used for his earlier cost avoidance calculations, this study further detailed estimated cost avoidance assuming a 9-day stay in Hawaii for routine ambulatory specialty evaluation. The presenting author estimated the cost avoidance during this 36 month window of evaluation to be over $\$ 3$ million [8].

\section{A Scalable Platform: Growth of PATH Beyond the Pacific}

Based on the success of PATH in the Pacific, Navy Medicine East and the US Navy Bureau of Medicine and Surgery (BUMED) launched the Health Experts onLine at Portsmouth (HELP) teleconsultation system in June 2014. HELP was a sub-module built on the existing PATH platform and housed on the server at TAMC, but used to provide improved access to care, and to reduce the need for travel to a tertiary care center, for medical providers stationed in Atlantic Fleet and Europe [3]. By 2017, the HELP platform was expanded to medical providers who were deployed to the Middle East, making it the largest reaching teleconsultation platform in the DoD virtual healthcare system [4].

A two-year review of HELP's return on investment (ROI) showed considerable savings (both tangible and intangible) to the Military Healthcare System for relatively minimal cost by decreasing the medical provider's reliance on medical evacuation, most often from a ship, in questionable cases utilizing a low bandwidth, asynchronous, and internet accessible teleconsultation system [6]. This review looked at the first two years of this relatively new platform, but with only 559 cases they were able to use a physician review panel with three independent reviewers to assess each case. The authors only considered it an avoided patient movement if all three reviewers concurred, which did happen in $80 \%$ of the HELP cases. They identified 50 cases where all three agreed that a movement of the patient was avoided through the use of asynchronous consultation. To evaluate return on investment, cost savings were compared to prevented patient transfer for any medical reason from either the continental US or outside the US, tangible savings (defined by prevented costs of flights, per diems, and in person consult visits) and intangible savings (time taken from work). Such savings were then compared to the upkeep costs to maintain the HELP system (startup costs, administrative cost, provider time cost). The total tangible savings was estimated to be $\$ 693,461$ (USD) for that 2 year review period, with an ROI of $81.15 \%$.

In 2017, a PATH/HELP pilot project brought the platform to military medical providers deployed to the Middle East. Comparing the window before introduction of 
the system to a window soon after the platform was introduced, there was an estimated $56 \%$ reduction in routine (non-emergency) medical evacuations from the Central Command area of operations [4]. Although there were many complexities comparing data of evacuations in a combat zone, the platform was widely accepted and is still utilized in the Middle East by deployed military providers over 3 years later.

\section{Summary}

For over two decades, Tripler Army Medical Center in Hawaii has served as a telemedicine hub, responding to the need for provider-to-provider teleconsultations by finding a solution that was well-received by both the originating site and distant site providers. The user-friendly web-based portal allowed clinicians to submit or respond to clinical consults in a manner that was easily incorporated to their daily workflow, and did not disrupt or complicate their routine patient care. The expansion of this platform, which started out serving a pediatric population, was driven by the request of users. It was well-integrated in the overseas military medical environment prior to restrictions being placed on travel due to a global pandemic, and is a model that could be applied to healthcare service in civilian settings as well. This low-bandwidth, secure system demonstrated increased access to care in a method that was both time-efficient and costsaving over the course of the 2 decades it has been used, and in various global settings $[1,5,6,8]$.

PATH has shown that an asynchronous, provider-to-provider model of teleconsultations can be widely accepted across many different populations and medical specialty areas. The user-driven growth of the platform speaks volumes to the importance of a system that is simple to use, yet provides high-yield information. By allowing a provider at a spoke location to ask a specialist detailed questions specific to their patient, it gave high quality, patient-specific actionable advice. In many respects, PATH has become such a regularly utilized tool for military healthcare providers in the Pacific that it might be generating clinical consults that a primary care provider might not have thought to ask, but knowing how quickly they could get a response decided to seek a brief, virtual "curbside" with a specialist.

PATH serves as a model of teleconsultation that could be applied to other, nonmilitary settings where travel distance hinders timely routine specialty care, or where the cost and complexity of synchronous teleconsultations might limit availability to specialty care. Future areas of review could include a comparison of PATH/HELP utilization before and after SARS-CoV-2 global pandemic, when global restrictions on movement into the US were placed for an extended period of time.

\section{Acknowledgements}

A heartfelt mahalo to the Tripler Army Medical Center Department of Pediatrics for over two decades of forward-leaning leadership in telehealth for the Pacific Rim, specifically the innovative thinking of Colonel (retired) Charles Callahan, Colonel (retired) Donald Person, and Colonel (retired) C. Becket Mahnke, and to the team that runs the Pacific Asynchronous TeleHealth (PATH) program and continues to carry their vision forward. 


\section{References}

[1] Callahan C, Malone F, Estroff D, Person D. "Effectiveness of an Internet-based store-and-forward telemedicine system for pediatric subspecialty consultation," Arch Pediatr Adolesc Med. 2005; 159:389393

[2] Malone F, Callahan C, Chan D, Sheets S, Person D. "Caring for children with asthma through teleconsultation: "ECHO-Pac, The Electronic Children's Hospital of the Pacific"," Telemed J E Health, $2004 ; 10,2: 138-46$

[3] Lin A, Cole J, Chin J and Mahnke C. "The Health Experts onLine at Portsmouth (HELP) system: Oneyear review of adult and Pediatric Asynchronous Telehealth Consultations," SAGE Open Med, Jan 2016.

[4] Nguyen C, Mbuthia J, Dobson C. "Reduction in Medical Evacuations from Iraq and Syria Following Introduction of an Asynchronous Telehealth System," Mil Med, 2020 Sep;1693-1699

[5] Mahnke C, Jordan C, Bergvall E, Person D, Pinsker J. "The Pacific Asynchronous TeleHealth (PATH) system: review of 1,000 pediatric teleconsultations," J Telemed E Health, 2011 Feb; 17(1): 35-39

[6] Lin A, Welstead B, Morey B, Mahnke C, Cole J, Johnston M. "Return on Investment Analysis of Health Experts onLine at Portsmouth: A 2-Year Review of the Navy's Newest Teleconsultation System," Mil Med, 2017 May; 185(5):1696-1701

[7] Wootton R, Liu J, Bonnardot L. "Assessing the quality of teleconsultations in a store-and-forward telemedicine network," Front Public Health. 2014 Jul;82(2)

[8] Miller M, Mbuthia J. "Updated Review and Three-Year Analysis of the Pacific Asynchronous TeleHealth System's Impact on Military Pediatric Teleconsultations," in The National Telehealth Research Symposium, Virtual, 2020 Nov;11-13 